\title{
Flucht und Migration bewegt Schüler/innen, Studierende und Lehrpersonen! Geographiedidaktik und Geographieunterricht für Menschenrechte und gegen Rassismus
}

\author{
spadberg@uni-wuppertal.de, Institut für Geographie und Sachunterricht, Bergische Universität Wuppertal \\ "*herbert.pichler@univie.ac.at, Institut für Geographie und Regionalforschung, Universität Wien \\ ***christiane.hintermann@univie.ac.at, Institut für Geographie und Regionalforschung, Universität Wien \\ ****stefan.baumann@phzh.ch, Fachbereich Geographie, Pädagogische Hochschule Zürich
}

Wir sind sehr besorgt um die Menschen, die aus Not in unsere Länder kommen. Wer vor Krieg und Verfolgung flüchtet, hat das völkerrechtlich verbriefte Recht auf Schutz. Der große Reichtum unserer Gesellschaften bietet mehr als genug Möglichkeiten, dieses Recht zu garantieren. Wenn auch viele, v. a. zivilgesellschaftliche Initiativen das Notwen(d)ige tun - von Freiwilligen aus den Ländern Europas an den Küsten von Lesbos bis zu jenen, die andere kostenlos in Deutsch unterrichten, so ist die Politik in Deutschland, der Schweiz und in Österreich in Richtung verstärkter Abgrenzung, Abschiebung und einer weiteren Einschränkung von Rechten der Geflüchteten unterwegs. Menschen, die vor Krieg und existentieller Not geflohen sind, sich dann mangels Alternative Schleppern, kaum seetauglichen Booten oder Wüstendurchquerungen ausliefern, werden etwa aktuell vom Territorium der EU in die Türkei abgeschoben. Damit in ein Land, das die Genfer Flüchtlingskonvention nur mit Einschränkungen unterschrieben hat und außereuropäischen Flüchtlingen keinen umfassenden Schutz gewährleistet (vgl. UNHCR 2016). Dass die Türkei als sicherer Drittstaat bezeichnet werden kann, wird von Hilfs- und Menschenrechtsorganisationen wie Pro Asyl, Human Rights Watch oder Amnesty International stark angezweifelt (vgl. Pro Asyl 2016).

Gleichzeitig wächst auf drastische Weise vor allem in Deutschland die Zahl fremdenfeindlicher und rassistischer Attacken auf die Geflüchteten. Das deutsche Bundeskriminalamt weist 1005 Angriffe auf Asylunterkünfte für das Jahr 2015 aus, davon 901 eindeutig aus dem rechtsradikalen Spektrum. Noch 2014 betrugen diese Zahlen nur ca. ein Fünftel (Spiegel online 2016). Auch in Österreich haben rechtsextreme, fremdenfeindliche, rassistische und antisemitische Tathandlungen laut jüngstem Verfassungsschutzbericht stark zugenommen. Sie stiegen von 2014 auf
2015 um mehr als die Hälfte auf 1156 (BMI 2015). In der Schweiz ist dem Nachrichtendienst des Bundes zufolge ein Gewaltpotenzial in der rechtsextremen Szene weiterhin vorhanden, die Lage wird aber derzeit insgesamt als weitgehend entspannt angesehen. Die angespannte Lage im Bereich Asyl habe aber das Potential, zu einer Lageverschärfung zu führen (vgl. NDB 2016).

Die Organisation Pro Asyl fasst folgende Forderungen zusammen, die wir ausdrücklich unterstützen (vgl. Pro Asyl 2016):

1. Gegen Hetze und Gewalt

2. Keine Einschränkung von Flüchtlingsrechten

3. Für Integration und Teilhabe von Anfang an

4. Für ein offenes Europa

Für das Erreichen dieser Ziele braucht es europaweit mehr Solidarität mit den Geflüchteten. Debatten um Obergrenzen lösen weder die Frage der Verteilung von Flüchtlingen noch mildern sie die Ursachen der Fluchtbewegungen. Weiters erachten wir den leichtfertigen Umgang hinsichtlich der Erklärung zu „sicheren Drittstaaten“"von Ländern wie der Türkei, Algerien oder Marokko als hochproblematisch (vgl. Brokmeier \& Erben 2016), unter anderem weil in diesen Ländern regelmäßig Minderheitenrechte aber auch beispielsweise Rechte von homo- und transsexuellen Menschen verletzt werden. Zudem wird durch die unzulässig generalisierende Konstruktion „sicheres Drittland“ die Einzelfallprüfung ausgesetzt. Wir verurteilen den Deal der Europäischen Union mit der Türkei und das Ziel, so einen ähnlichen Status quo wie vor der so genannten „Flüchtlingskrise“ wieder herzustellen. Es darf nicht das Ziel europäischer Politik sein, die „Festung Europa“ durch Verunmöglichung einer legalen Beantragung von Asyl erneut so auszugestalten, dass Flüchtende gezwungen werden, 
immer riskantere Routen in Kauf zu nehmen, weiter ihr Leben zu riskieren und diese vielfach dabei zu verlieren. Vielmehr muss es darum gehen, internationale Rechte zu achten, Menschen in Seenot zu retten und ihre Rechte individuell zu prüfen. Mehr noch: Die humanitäre Katastrophe an den Außengrenzen der Europäischen Union muss mit legalen und sicheren Einreisemöglichkeiten proaktiv verhindert werden um Menschenrechte zu garantieren. Die durch verstärkte Sicherung der Außengrenzen vorangetriebene Abschottung Europas sowie die dadurch billigend in Kauf genommenen Folgewirkungen müssen als menschenverachtend und Menschenrechte verletzend bezeichnet werden.

Als Geographiedidaktiker/innen und -lehrer/innen stehen wir für die Menschenrechte ein. Wir rufen dazu auf, Seminarveranstaltungen und Unterricht zu Menschenrechten, Migration und Rassismuskritik anzuregen und anzuleiten. Dazu stellen wir im Folgenden einige Fakten und Materialien zusammen, die bei diesem Vorhaben unterstützen mögen. Wie die Seminarstunden und der Unterricht konkret stimmig an die lokalen Verhältnisse und die jeweilige Lerngruppe angepasst werden soll, kann und wird die jeweilige Lehrperson am besten selbst beurteilen. Daher legen wir das Schwergewicht in diesem Beitrag auf orientierende Gedanken, Hintergrundinformationen und didaktische Grundüberlegungen. Zusätzlich finden sich abschließend einige Verweise auf konkrete Unterrichtsideen.

\section{$1 \quad$ Migration - was ist unverändert?}

Die Ursachen der Migration sind die gleichen, die seit Jahrzehnten zu verzeichnen sind:

\subsection{Kriege, oft unter Beteiligung ehemaliger Kolonialmächte}

Syrien, Afghanistan, Libyen und Eritrea sind vier zahlenmäßig sehr bedeutsame Herkunftsländer aktuell Flüchtender. In allen Fällen ist Krieg oder Bürgerkrieg die Ursache und westliche Staaten auf Grund ihrer kolonialen Vergangenheit oder aktueller geopolitischer und wirtschaftspolitischer Interessen Partei in den Auseinandersetzungen. In Syrien und Libyen wurden etwa jahrzehntelang Diktaturen durch Hofierung westlicher Regierungen gestützt, in Afghanistan die Taliban durch die USA gegen die UdSSR aufgerüstet. Auch der "Islamische Staat“, eine weitere Ursache für Flucht aus Syrien und dem Irak, wird indirekt über Waffenlieferungen an Saudi-Arabien von der BRD sowie durch Ölkäufe multinationaler Konzerne unterstützt (Napoleoni 2015).

\subsection{Entzug der wirtschaftlichen Lebensgrundla- gen}

Die globale Wirtschafts(un)ordnung produziert kontinuierlich wachsende ökonomische Ungerechtigkeiten. Beispiele sind die Ausweitung der industriellen Fischerei vor Westafrika oder der Export von Überschüssen der europäischen Nahrungsmittelproduktion in afrikanische Staaten und damit die Zerstörung lokaler Märkte in diesen Sektoren.

Dass diese (Zer)Störung lokaler und regionaler Wirtschaftskreisläufe und damit der Lebensgrundlage vieler Menschen wesentlich zum Aufbau von Migrationspotenzialen beiträgt, ist seit dem Weltsystemansatz von Immanuel Wallerstein (1974) bekannt und theoretisch untermauert. Verallgemeinernd kann festgestellt werden, dass die konsum- und wachstumsorientierte, ressourcenverschwendende Lebensweise der Mehrheit der Menschen in den Ländern des globalen Nordens die globalen natürlichen Lebensgrundlagen beeinflusst und so neue bzw. zusätzliche Fluchtgründe v. a. für die bäuerliche Bevölkerung im globalen Süden befördert.

\subsection{Migration kann illegalisiert werden, aber nicht aufgehalten}

Solange die Ursachen der Fluchtbewegungen nicht behoben sind, und solange Flüchtende auch in den Auffanglagern in Nachbarstaaten keine Perspektive für sich und ihre Kinder erkennen können, werden Menschen, die nichts zu verlieren haben, versuchen, ihr Überleben durch den Weg nach Europa zu sichern und Versprechungen von Schleppern glauben, bzw. aus ihrer Perspektive: Fluchthelfer bezahlen. Sowohl bei der Ausreise als auch bei der Durch- und Einreise ist Migration nur mit enormen finanziellen Kosten und unter Einsatz militärischer Mittel nur sehr bedingt steuerbar (Eule 2016). Die Geschichte des Ausbaus und der Militarisierung der Grenze zwischen Mexiko und den Vereinigten Staaten lehrt, dass Migration durch die Errichtung und Befestigung von Grenzzäunen nicht verhindert werden kann (Koslowski 2011). Die Illegalisierung von Migration führt jedoch zu mehr sogenannten illegalen Grenzübertritten, zu gefährlicheren Routen der Flüchtenden und zu Mehreinnahmen für Schlepper. Dadurch verschlechtern sich das Leben und die (Über-)Lebensmöglichkeiten der Flüchtenden erheblich.

\subsection{Die globale Verteilung der Migration ist nahezu unverändert.}

Die UN-Flüchtlingsorganisation UNHCR verzeichnet derzeit 60 Millionen Flüchtende weltweit. Zwei 
Drittel davon leben als Binnenflüchtlinge im eigenen Land, viele zusätzlich in den Nachbarländern. 86 Prozent der 60 Millionen leben in so genannten Entwicklungsländern. So beherbergt allein Libanon - ein Land mit vier Millionen Einwohner/innen - Zahlen des UNHCR zufolge mehr als eine Million syrische Flüchtlinge. Die wenigsten erreichen Europa, Nordamerika oder Australien. Zu beobachten ist auch, dass der Fluchterfolg offenbar mit dem soziökonomischen Status der Flüchtenden korreliert. Wer besser informiert war und sich (mehr) Schlepper leisten konnte, kam zumindest vor der Schließung der Balkanroute schneller und weiter voran.

\subsection{Das Sterben an den Außengrenzen der „Fes- tung Europa“"}

Fast täglich ertrinken Menschen in oder nahe europäischer Gewässer, derzeit vor allem an den Grenzen der Türkei mit Griechenland und auf dem Weg von Nordafrika nach Lampedusa oder anderen südeuropäischen Küsten. Seit Jahren sterben zahllose Flüchtende beim Versuch, mit untauglichen Booten das Mittelmeer zu überqueren um auf europäisches Territorium zu gelangen. Zwischen 2000 und 2013 rechnet etwa Le Monde diplomatique mit mehr als 23.000 Todesopfern an der europäischen Außengrenze (23.3.2014). Die Nachrichten von 71 erstickten Flüchtlingen im Frachtraum eines Kühl-LKWs bei Parndorf in Österreich im Sommer 2015 oder die Bilder der an den Strand gespülten jungen Aylan im Januar 2016 haben deutlicher als bisher dieses alltägliche Leid in das Bewusstsein der Öffentlichkeit in Österreich, Deutschland und der Schweiz getragen. Gleichzeitig haben diese emotionalisierenden Einzelereignisse nicht zu einer nachhaltigen Stimmungs- und Politikveränderung beigetragen.

Die italienische Initiative „Mare Nostrum“, die viele Flüchtende vor dem Ertrinken rettete, wurde bereits 2014 durch die Nicht-Unterstützung der EU beendet, nachdem Italien die EU ersucht hatte, die Kosten für die Aktion zu übernehmen, die es selber nicht mehr tragen könne. Vor allem Deutschland hatte dies mit der Begründung abgelehnt, Mare Nostrum sei für die Flüchtenden eine „Brücke nach Europa“ (Deutschlandfunk 2014; Spiegel online 2014; NZZ 2014).

Statt legale und sichere Zugänge in die EU zu schaffen, wird die Grenzschutzagentur Frontex ausgebaut und die Politik gegen Schlepper verschärft, deren Geschäftsgrundlage genau in der Ausweitung der Abschottung und Militarisierung der Außengrenzen der EU besteht. Nach dem politischen Abkommen mit der Türkei zur „Rücknahme“ geflüchteter Menschen aus Griechenland ist eine Verschärfung dieses politischen Kurses zu erwarten. Als Ungarn 2015 begann einen Grenzzaun zu bauen, wurde dies noch von vielen europäischen Regierungen scharf kritisiert. Durch die Schließung der sogenannten „Balkanroute“, auch auf Druck der österreichischen Bundesregierung, über Mazedonien und Slowenien, verlagern sich derzeit die Schlepperrouten wieder auf gefährlichere und längere Wasserwege von nordafrikanischen Küsten nach Europa.

\section{Migration nach Österreich, in die Schweiz und die BRD - was ist neu?}

Tatsächlich neu an der Flüchtlingsbewegung ist, dass Flüchtende 2015 in beachtlicher Zahl lebend Mitteleuropa erreichten. Es gelang ihnen, die Schengen-Außengrenzen einerseits in Griechenland zu überqueren und andererseits von Afrika kommend aus Italien weiter nach Norden zu reisen. Nach den - scheinbar vorübergehenden - Ursachen dieser Veränderung wurde in der öffentlichen Debatte wenig gefragt, dabei sind sie leicht nachvollziehbar (vgl. Schiffer-Nasserie 2015). Die österreichische Tageszeitung Der Standard berichtete z. B. bereits im Dezember 2014, dass das Welternährungsprogramm der UNO (WFP) die so genannte Hungerhilfe (sic!) an 1,7 Millionen Flüchtlinge in den Nachbarstaaten Syriens einstellen musste, nachdem wichtige Geberländer ihren Verpflichtungen nicht nachgekommen waren (Der Standard 2014). Die drastische Unterfinanzierung der Hilfe vor Ort hatte und hat unmittelbare Auswirkungen auf die Zahl der Menschen, die den gefährlichen Fluchtweg Richtung Europa antreten.

Im Jahr 2015 gewann in Griechenland SYRIZA die Wahlen und verhandelte über Monate mit der Troika der EU über finanzielle Entlastungen. Diese zu erreichen gelang der griechischen Regierung nicht. De facto ließ sie ab Sommer jedoch Flüchtende weiter nach Norden reisen - was sie als Staat an der SchengenAußengrenze eigentlich nicht darf (vgl. Amtsblatt der EU 2016). So etablierte sich die Balkanroute.

Wie bereits erwähnt, weigerte sich die EU Italien gegenüber die Aufrechterhaltung des Programms "Mare Nostrum" mit zu finanzieren. Wenig später tauchten in Hamburg Geflüchtete aus Lampedusa kommend auf, die offensichtlich von der italienischen Regierung mit Bahntickets ausgestattet waren - gewissermaßen ein lebendiger Gruß der italienischen Regierung (vgl. Schiffer-Nasserie 2015).

Durch die in weiterer Folge verstärkten Kontrollen an den EU-Außengrenzen und das Schließen der Balkanroute war die Zahl der neuen Asylanträge in Österreich und Deutschland seit Jahresbeginn 2016 rückläufig. Die erwartbare Verlagerung der Fluchtrouten ließ Österreich seit Mai 2016 Vorbereitungen zu 
permanenten Kontrollen an der Brennergrenze treffen, was zu heftigen diplomatischen Verstimmungen mit Italien führte. Keine der bislang getroffenen politischen Maßnahmen rührt jedoch an den konkreten Fluchtursachen und -gründen, keine der Maßnahmen verbessert die Lebenssituation der Geflüchteten. Damit sind die Maßnahmen bislang nicht zur Lösung der Situation geeignet.

\section{Was wird öffentlich gesagt und getan?}

Das hier Zusammengestellte erhebt keinen Anspruch auf Vollständigkeit und seine Aktualität wird zum Zeitpunkt der Veröffentlichung notwendigerweise bereits gelitten haben. Diese Zusammenstellung soll aufzeigen, vor welchen Hintergründen und wie sich die politische Debatte und die mediale Vermittlung der so genannten „Flüchtlingskrise“ abspielt und verändert.

Als sich Flüchtende im Sommer 2015 vermehrt über Griechenland nach Europa aufmachen, ruft Angela Merkel eine Willkommenskultur aus: „Wir schaffen das". Wie es zu ihrem Sinneswandel kam, der sie plötzlich dazu veranlasste, diese klaren Verstöße gegen das Schengener Abkommen zu dulden, ist ein Punkt, der wenig debattiert wird (vgl. Schiffer-Nasserie 2015). Hier sei nur angedeutet, dass die deutsche Wirtschaft stark exportorientiert aufgestellt ist und an geschlossenen Grenzen in Europa keinerlei Interesse hat.

Neben der widersprüchlichen Politik der deutschen Bundesregierung und dem vielfältigen ehrenamtlichen Engagement zur Unterstützung der Geflüchteten stehen gleichzeitig von der Sozialdemokratie über ÖVP, CDU, SVP und FPÖ bis in die extreme Rechte (auch weit über den deutschsprachigen Raum hinaus) skeptische bis offen rassistische Aussagen und Forderungen zur Frage des Umgangs mit Flüchtenden. Extremer Ausdruck dieser menschenverachtenden Grundhaltungen war etwa das öffentliche Nachdenken der Spitzenkandidatin der „Alternative für Deutschland“ (AfD) über den Gebrauch von Schusswaffen gegen Flüchtende an der deutschen Grenze. Im Karnevalszug einer ostdeutschen Stadt fährt im Februar 2016 eine Attrappe eines Wehrmachtspanzers mit der Aufschrift „Asylabwehr“ (Focus online 2016). Tausende demonstrieren in der BRD seit Monaten gegen die angebliche „Islamisierung des Abendlandes“. Der Bayrische Ministerpräsident Horst Seehofer redet am politischen Aschermittwoch von einer „Herrschaft des Unrechts" im Hinblick auf die 2015 geöffneten deutschen Grenzen. Die im Bund mitregierende SPD und Bundespräsident Gauck sprechen wie selbstverständlich davon, dass mit Blick auf das laufende Jahr 2016 die „Belastungsgrenzen“ des Landes im Hinblick auf die Aufnahme weiterer Geflüchteter zu beachten sei. Auch in mitte-links Kreisen in der Bevölkerung wird über Aufnahmezahlen für 2016 geredet, als gäbe es keine Genfer Konvention und keine internationalen Verpflichtungen hinsichtlich etwa der Rettung von Menschen in Seenot und des Schutzes geflüchteter Menschen aus Kriegsgebieten.

Vielfach wird argumentiert, die Kommunen seien an ihren Grenzen angelangt, was die Aufnahme von Geflüchteten angeht. Was in Deutschland hingegen kaum Erwähnung findet, ist, dass die Kapazitäten zur Aufnahme von Geflüchteten nicht an die zu erwartenden Zahlen angepasst wurden. Im Gegenteil dazu wurden in Deutschland in den letzten Jahren parallel zur „Lösung“, die Flüchtenden an den Außengrenzen aufzuhalten, in den Kommunen tendenziell Kapazitäten abgebaut (Schiffer-Nasserie 2015).

In der Schweiz wurde im Februar 2016 über die Abschaffung von Grundrechten für Migrantinnen und Migranten auch der zweiten Generation abgestimmt. Damit stand ein wesentliches Merkmal des Rechtsstaats, „Jeder Mensch ist vor dem Gesetz gleich“" zu Debatte. In dieser Phase veröffentlicht die NZZ am Sonntag den Artikel eines deutschen Historikers, in dem dieser den Untergang des römischen Reiches durch die offenen Grenzen und die Einwanderung zu belegen meint. Auch jenseits der offensichtlichen sachlichen Schwäche der Argumentation - es konnte bis in jüngsten Zeit, allein aus technischen Gründen, kaum im heutigen Sinne befestigte Grenzen geben - war im Vorfeld der Zürcher Veröffentlichung der deutschen CDU-nahen Konrad-Adenauer-Stiftung die Publikation dieses Artikels in der aktuellen Debatte zu heikel.

Ein großer Lichtblick in der deutschsprachigen Debatte in der Schweiz ist der Sieg der Gegner/innen in dieser Abstimmung - herbeigeführt durch das engagierte Auftreten der Operation Libero: junge Liberale, die die Politbühne erstmals betraten, fremdenfeindliche Parolen konsequent mit pointierten Argumenten entkräfteten, damit die prognostizierte $60 \%$-Zustimmung innert zwei Monaten in eine $60 \%$-Ablehnung wandelten und schließlich das Polit-Establishment am Tag der Abstimmung hörbar zum Aufatmen brachte.

Auf politischer Ebene steht in der Schweiz zur Debatte, wie viele Asylbewerber/innen aufgenommen werden „können“. Im Alltag bereiten sich aber Bund, Kantone und Gemeinden darauf vor, Asylbewerber/ innen eine Unterkunft zur Verfügung zu stellen, solange sie keinen negativen Entscheid erhalten haben. So werden aktuell in vielen Gemeinden Zivilschutzunterkünfte für die temporäre Aufnahme vorbereitet. Seit absehbar wurde, dass die Balkanroute "geschlossen" werden soll, wurde in der Schweiz ein so genann- 
tes „Notfallkonzept“ verabschiedet, um eine mögliche Zunahme der Asylanträge an der Grenze zu Italien zu bewältigen. Als „Notfall“ werden drei unterschiedliche Szenarien definiert, die sich je nach Zahl der Asylgesuche innerhalb eines bestimmten Zeitraumes unterscheiden und zwischen 10000 Asylanträgen innerhalb von drei Monaten und 30000 Asylgesuchen innerhalb weniger Tage schwanken. Der „Notfallplan“ soll sicherstellen, dass alle Asylsuchenden registriert, untergebracht und betreut werden können, erlaubt es den Behörden aber auch individuelle Sicherheitsüberprüfungen durchzuführen und den Grenzschutz zu verstärken. Bund, Kantone und Gemeinden arbeiten bei der Suche und Bereitstellung geeigneter Asylunterkünfte zusammen (NZZ 2016). Im Juni 2016 wird zudem über ein neues Asylgesetz abgestimmt, das zum Ziel hat, die Asylverfahren zu beschleunigen. In einem über zwei Jahren dauernden Pilotbetrieb wurden die neuen Verfahren erfolgreich getestet, weshalb bis auf die SVP alle großen politischen Parteien und auch NGO's wie z. B. die Flüchtlingshilfe dahinterstehen. Ende Mai 2016 wird davon ausgegangen, dass sich die politische Rechte mit ihrem Referendum nicht wird durchsetzen können.

Österreich reagiert auf die ersten ankommenden Flüchtlingszüge im Sommer 2015 zunächst unterstützend: Exekutive, karitative Organisationen und zahllose freiwillige Helfer/innen bilden eine Koalition zur Erstversorgung und Unterstützung der Flüchtlinge. Die Österreichische Bundesbahn verzichtet auf Fahrscheinkontrollen, zahllose Bustransfers nach Deutschland werden organisiert, Hunderttausende werden an die deutsche Grenze transportiert. Die anfänglichen Bilder von Flüchtlingen, die bei ihrer Ankunft am Wiener Westbahnhof von Applaus der Helfer/innen empfangen werden, verändern sich gemeinsam mit der medialen und politischen Debatte. Nun steht die Diskussion um das Erreichen einer „Belastungsgrenze" im Mittelpunkt, mehrere Gemeinden wehren sich aktiv gegen die Errichtung von Flüchtlingsunterkünften. Interessierte Leser/innen beobachten zudem, wie ausgehend von Gratiszeitungen und Boulevardmedien Flüchtende und Asylwerber/innen in der Berichterstattung zunehmend mit Kriminalität in Verbindung gebracht wurden. Es werden von Seiten der Politik Schritte unternommen, wonach Asylwerber/innen in Kursen über in Österreich geltende Werte in Kenntnis gesetzt werden sollen. Eine klare Vermittlung der gesetzlichen Bestimmungen, der Rechte, Pflichten und Verbote, wie sie für alle Menschen in unseren Staaten im Rahmen politischer Bildung wünschenswert ist, wäre möglicherweise weniger kritisierbar und effektiver.

Das Bundesland Wien nimmt deutlich mehr Flüchtlinge auf als im Verteilungsschlüssel zwischen
Bund und Ländern vorgesehen, vor allem weil andere Bundesländer ihren Verpflichtungen nicht nachkommen. Der Wiener Bürgermeister Häupl setzt sich bei Gemeinderatswahlen im Herbst 2015 mit seiner an Menschenrechten orientierten Linie in der Flüchtlingsfrage gegen die flüchtlingsfeindliche Kampagne der FPÖ durch.

Die österreichische Bundesregierung verfolgt das Ziel der Eindämmung der „Balkanroute“ und erreicht durch Einwirken auf Länder wie Mazedonien oder Slowenien die jeweilige Errichtung und Kontrolle von Grenzzäunen. Weiter beschließt die SPÖ-ÖVPKoalition menschenrechtswidrig eine Obergrenze von 37500 Asylanträgen für 2016 und verschärft - entgegen den Grundsätzen des Schengener Abkommens - die Grenzkontrollen an den Übergängen zu Slowenien und Italien. Mehr als 25 Jahre nachdem der „Fall des Eisernen Vorhanges" bejubelt wurde, errichtet Österreich an seinen Außengrenzen abschnittsweise Grenzzäune. Österreich hat von 2015 bis 2016 im Vergleich zu Größe und Bevölkerungszahl und verglichen mit anderen EU-Staaten überdurchschnittlich viele Flüchtende aufgenommen. Dennoch beherbergt ein Drittel der österreichischen Gemeinden im Frühjahr 2016 keine einzige Asylwerberin, keinen einzigen Asylwerber.

Im österreichischen Bundespräsidentschaftswahlkampf 2016 spielt das Flüchtlingsthema eine dominierende Rolle. Norbert Hofer von der FPÖ (Ehrenmitglied der schlagenden Burschenschaft Marko-Germania), der den 1. Wahlgang deutlich für sich entscheiden konnte und in der Stichwahl fast 50 Prozent der Stimmen erhielt, bezeichnete Flüchtlinge im Wahlkampf als „Invasoren“ und spricht sich u.a. für eine spezielle Sozialversicherung ohne Arbeitslosenversicherung für ausländische Arbeitskräfte aus (Der Standard 2016). Ende April beschließt der österreichische Nationalrat eine weitere Verschärfung des Asylgesetzes, die es u. a. ermöglicht, Asylantragsteller/ innen an der Grenze abzuweisen, wenn die Regierung im Einvernehmen mit dem Hauptausschuss des $\mathrm{Na}$ tionalrates die innere Sicherheit und die öffentliche Ordnung in Österreich bedroht sieht (vgl. UNHCR 2016). Ebenfalls im April 2016 spricht der deutsche Innenminister davon, dass die Grenzkontrollen nach Österreich wieder eingestellt werden sollen. Der Status Quo der Festung Europa mit militärisch gesicherten Außengrenzen soll die Reisefreiheit innerhalb des Kontinents wieder uneingeschränkt erlauben.

Die fehlende europäische Solidarität bei der Aufnahme oder Verteilung von Flüchtlingen ist in der aktuellen Situation besonders augenscheinlich. Der Beschluss der EU-Kommission vom September 2015 160000 Geflüchtete aus Griechenland und Italien auf andere EU-Staaten zu verteilen, stieß auf heftigen Wi- 
derstand vor allem von Seiten ost- und ostmitteleuropäischer Mitgliedsstaaten und harrt immer noch der Umsetzung. Neu ist die mangelnde Übernahme von Verantwortung vieler europäischer Regierungen im Bereich Asylpolitik jedoch nicht. Ceuta, Melilla oder Lampedusa stehen beispielhaft dafür, wie gering das Interesse an einer gesamteuropäischen Politik war, solange die eigenen nationalstaatlichen Grenzen davon nicht berührt wurden.

\section{Wie kann Geographiedidaktik und Geo- graphieunterricht reagieren? Ein Aufruf: Geographiedidaktik und Geographieunterricht für Menschenrech- te und gegen Rassismus!}

\subsection{Die Geographie- und GW-Didaktik tragen Verantwortung}

Der Geographie im deutschsprachigen Raum erwächst im thematischen Kontext auch daraus eine besondere Verantwortung, da sie als Wissenschaft das Entstehen und die Ausbreitung des Nationalsozialismus mit seiner Rassenideologie und seinen Raumansprüchen unkritisch bis zustimmend begleitet und zum Teil argumentativ unterstützt hat (Schultz 2001). Rassismus in Schulbüchern ist auch heute noch vereinzelt nachweisbar (Mönter \& Schiffer-Nasserie 2007), ausschließende Diskurse sind in Schulbuchtexten eher die Regel als die Ausnahme (Hintermann 2010). Daher sind Schweigen und Enthaltung keine Optionen.

Die aktuellen Geschehnisse und Debatten über Flucht, Migration und ihre Folgewirkungen für Herkunfts-, Zielregionen und Gesellschaften sind ein Auftrag an einen vernetzenden und mehrperspektivischen Geographieunterricht. Damit wird es zur Aufgabe der Schulgeographie und der wirtschaftlichen Bildung, den Prozess der Globalisierung, zu dessen Phänomenen auch Migration gehört, in all seinen Widersprüchen erkennbar und damit kritisierbar und gestaltbar zu machen. Dazu gehören u. a. die Zusammenhänge zwischen den heutigen und einstigen kolonialen Weltwirtschaftsstrukturen, die ökonomischen und ökologischen Auswirkungen von Freihandelszonen, die Folgen der Ausbeutung der Rohstoffe, der anthropogenen Verknappung von fruchtbaren Böden und Trinkwasser sowie des Klimawandels. Weiters ist es Ziel von geographischer und wirtschaftlicher Bildung, diese Zusammenhänge und ihre Auswirkungen nicht nur global zu verorten, sondern auch Interessen und Verantwortung in den Machtgefügen auf verschiedenen Maßstabsebenen zu erörtern und historische Verantwortlichkeiten zu benennen. Nur so werden unsere Fächer ihrem Auftrag gerecht, mehrperspektivisch und politisch bildend wirksam zu sein. Dazu gehört zwingend, die Einzelne und den Einzelnen über die eigene Verantwortung und die Interventionsmöglichkeiten zu informieren und in der Selbstwahrnehmung als politisches Subjekt zu stärken (vgl. Gardi et al. 2016).

\subsection{Didaktische Orientierung}

Folgende didaktischen Überlegungen können die Basis einer Auseinandersetzung im Geographie (und Wirtschaftskunde) Unterricht bilden. Ausgehend von technischen, praktischen sowie kritisch-emanzipatorischen Vermittlungsinteressen (Vielhaber 1999) lassen sich jeweils begründbare Unterrichtsziele und Fragestellungen entwickeln, die für Lehrpersonen handlungsleitend sein können. Wir rufen Lehrende, Studierende sowie Schüler/innen dazu auf gemeinsam einen politisch bildenden Geographie (und Wirtschaftskunde) Unterrichts zu gestalten, der es ermöglicht,

- Zusammenhänge zwischen unserer Wirtschaftsweise und globalen Fluchtursachen zu erkennen und zu benennen,

- aktuelle politische und mediale Diskurse bezüglich Flüchtlingen in unseren Ländern kritisch zu reflektieren und darin mit begründeten Argumenten und Zahlen einzugreifen (Pro Asyl 2014),

- die eigenen potentiell alltagsrassistischen Denkund Redeweisen zu prüfen (Mecheril 2016),

- die Perspektive der Geflüchteten einzunehmen und so verstehen zu können, dass Menschen nur aus guten und in aller Regel nachvollziehbaren Gründen Lebensgefahr auf sich nehmen, um eine äußerst ungewisse Zukunft möglich zu machen (Sasse 2016),

- dass Migrantinnen und Migranten und Geflüchtete selbst zu Wort kommen (Selbstrepräsentation) und nicht nur als „Objekte“ im Unterricht besprochen werden,

- die Migrationsgeschichten der eigenen Familien zu erforschen, um zu erkennen, wie über mehrere Generationen zurück Migration Teil der eigenen Familiengeschichte sein kann,

- Migration als eigene Zukunftsoption zu reflektieren sowie die Motive einer potentiellen eigenen Emigration mit jenen von Migrantinnen und Migranten und Flüchtenden zu vergleichen,

- gegen Rassismus von privater und institutioneller Seite einzutreten,

- die Unteilbarkeit der Menschenrechte in all ihren Konsequenzen zu reflektieren,

- Diversität als kulturelle, gesellschaftliche und ökonomische Chance sowie als Bereicherung wahrzunehmen. 
Nehmen wir die aktuelle Situation zum Anlass, die Themen Flucht, Migration und Diversität über die demographische Perspektive und den Kosten-NutzenDiskurs hinaus als komplexe Phänomene zu diskutieren. Schulen wir damit einen mehrperspektivischen und kritischen Blick auf gesellschaftliche Entwicklungen. Erschließen wir die Komplexität der Thematik auch ausgehend von lebensweltlichen Erfahrungen und Bezügen unserer Schüler/innen, um diese im mehrfachen Wortsinn zu bewegen. Ermöglichen wir auf dieser Basis Bildungsprozesse und berichten darüber öffentlichkeitswirksam innerhalb und außerhalb der Schule.

\section{Literatur}

\section{Literatur und Materialien zum Hintergrund}

Amtsblatt der EU (2016): Verordnung 2016/399 des Europäischen Parlaments und des Rates vom 9. März 2016 über einen Gemeinschaftskodex für das Überschreiten der Grenzen durch Personen (Schengener Grenzkodex).

http://eur-lex.europa.eu/legal-content/DE/TXT/PDF/?uri $=$ CELEX:32016R0399\&from=DE (28.05.2016).

BMI - Bundesministerium für Inneres (2015): Verfassungsschutzbericht 2015. http://www.bmi.gv.at/cms/BMI_ Verfassungsschutz/Verfassungsschutzbericht_2015.pdf (28.05.2016).

Brokmeier, B. \& F. Erben (2016): Zuwanderung. Flucht und Asyl im Fokus politischer Bildung. In: Journal für Politische Bildung 2/2016, 32-39.

Büsing, M. (o. J.): Karte der Toten im Zusammenhang mit Flucht in Europa und an seinen Grenzen. http://15years. morizbuesing.com (28.05.2016).

Der Standard (2014): UNO stellt Hungerhilfe für 1,7 Millionen syrische Flüchtlinge ein, 01.12.2014. http://derstandard.at/2000008848109/UNO-stelltHungerhilfe-fuer-17-Millionen-syrischen-Fluechtlingeein (28.05.2016).

Der Standard (2016): Hofer: AMS soll an Migranten keine Jobs vermitteln, 18.04.2016. http://derstandard. at/2000035127392/Hofer-AMS-soll-an-Migrantenkeine-Jobs-vermitteln (28.05.2016).

Deutschlandfunk (2014): Italien beendet Rettungsaktion „Mare Nostrum“, 31.10.2014. http://www.deutschlandfunk.de/fluechtlingeitalien-beendet-rettungsaktion-mare-nostrum.1818. de.html?dram:article_id=301920 (28.05.3016).

Eule, T. (2016): Können wir Migration steuern? Erfahrungen aus der internationalen Migrationsrechtspraxis. Vortrag im Rahmen der Reihe: Flucht, Migration, Integration an der Universität Bern. http://www.migration.unibe. ch/videos/01_maerz_2016 (28.05.2016).

Fischer, T. (2016): Unser Sexmob. In: Zeit Online, „Fischer im Recht", 12. 01.2016. http://www.zeit.de/gesellschaft/ zeitgeschehen/2016-01/sexmob-koeln-kriminalitaetstrafrecht-fischer-im-recht (28.05.3016).
Focus online (2016): Panzerattrappe «Asylabwehr»Diese Motivwagen sorgten beim Karneval für Entrüstung, 09.02.2016. http://www.focus.de/politik/deutschland/ panzerattrappe-asylabwehr-diese-motivwagen-sorgtenbeim-karneval-fuer-entruestung_id_5270119.html (28.05.2016).

Gardi, N., U. Lingen-Ali \& P. Mecheril (2016): Politische Bildung unter Bedingungen von Flucht und globaler Ungleichheit. In: Journal für Politische Bildung 2/2016, 8-16.

Hintermann, C. (2010): Schulbücher als Erinnerungsorte der österreichischen Migrationsgeschichte - eine Analyse der Konstruktion von Migrationen und Migrant/ innen in GW-Schulbüchern. In: GW-Unterricht 119, 3-18. http://www.gw-unterricht.at/images/ pdf/gwu_119_003_018_fachdidaktik_hintermann_ migration.pdf (28.05.2016).

Koslowski, R. (2011): The Evolution of Border Controls as a Mechanism to Prevent Illegal Immigration. Washington, DC: Migration Policy Institute. http:// www.migrationpolicy.org/research/evolution-USborder-controls-illegal-immigration (28.05.2016).

Labor für kritische Migrations- und Grenzregimeforschung. http://kritnet.org (28.05.2016).

Manach, J.-M. (2014): Ces gens-là sont morts, ce ne sont plus des migrants. In: Le Monde diplomatique. 31.03.2014. http://www.monde-diplomatique.fr/ carnet/2014-03-31-morts-aux-frontieres (28.05.2016).

Mecheril, P., M. do Mar Castro Varela, I. Dirim, A. Kalpaka \& C. Melter (2010): Migrationspädagogik. WeinheimBasel: Beltz Verlag.

Mecheril, P. (2016): Die Gastrede von Paul Mecheril. In: Weserkurier, 14.01.2016. http://www.weser-kurier. de/bremen/bremen-politik-wirtschaft_artikel,-DieGastrede-von-Paul-Mecheril-_arid,1291009.html (28.05.2016).

Messerschmidt, A. (2016): Nach Köln - Sprechen über Sexismus und Rassismus. In: Zur Ethnisierung der Genderdebatte. Überblick. Informations- und Dokumentationszentrum für Antirassismusarbeit in NRW (IDA-NRW), 1 (16), 7-10. http://www.idanrw.de/publikationen/ueberblick/ueberblick.html (28.5.3016).

Mönter, L.O. \& A. Schiffer-Nasserie (2007): Antirassismus als Herausforderung für die Schule. Von der Theoriebildung zur praktischen Umsetzung im geographischen Schulbuch. Frankfurt a. M. u. a.: Lang.

Napoleoni, L. (2015): Die Rückkehr des Kalifats. Der Islamische Staat und die Neuordnung des Nahen Ostens. Zürich: Rotpunktverlag

NZZ-NeueZüricher Zeitung (2014): «Mare Nostrum» Von einer Erfolgsgeschichte zum Desaster, 14.10.2014. http:// www.nzz.ch/international/von-einer-erfolgsgeschichtezum-desaster-1.18398148 (28.05.2016).

Pro Asyl (2014): Pro Menschenrechte, contra Vorurteile. Fakten und Argumente zur Debatte über Flüchtlinge in Deutschland und Europa. Frankfurt a.M. http://www.proasyl.de/fileadmin/fm-dam/q 
PUBLIKATIONEN/2014/Broschuere_Pro_MR_ Contra_Rassismus_Web.pdf (28.05.2016).

Pro Asyl (2016): Rassismus bedroht Flüchtlinge und eine offene Gesellschaft. http://www.proasyl.de/wp-content/ uploads/2015/12/Pro_Asyl_Faltblatt_Aufstehen_gegen_ Maerz_2016.pdf (18.04.2016).

Sasse, E. (2016): My Escape (Film aus Handydokumenten Geflüchteter). http:/www1.wdr.de/mediathek/ video/sendungen/wdr-dok/video-my-escape--meineflucht-100.html (18.04.2016).

Schiffer-Nasserie, A. (2015): Acht Thesen zu „Flüchtlingskrise“ und „Willkommenskultur“. In: Flucht - Ursachen, Hintergründe, Konsequenzen. Überblick. Zeitschrift des Informations- und Dokumentationszentrums für Antirassismusarbeit in Nordrhein-Westfalen, 21 (4), 3-8. http://www.idanrw.de/publikationen/ueberblick/ueberblick.html (18.04.2016).

Schultz, H.-D. (2001): „Die geschlossene Nation marschiert." Der Erdkundeunterricht im Dritten Reich zwischen Raum und Rasse. In: Dithmar, R. \& W. Schmitz (Hrsg.): Schule und Unterricht im Dritten Reich. Ludwigsfelde ( = Interdisziplinäre Forschung und fächerverbindender Unterricht Bd 7), 231-267.

Spiegel online (2014): Streit über Flüchtlinge: Rom rettet, Berlin blockiert, 09.07.014. http://www.spiegel.de/ politik/ausland/eu-und-italien-streiten-um-fluechtlingeund-mare-nostrum-a-979917.html (28.05.2016).

UNHCR - United Nations High Commissioner for Refugees (2016): UNHCR zur EU-Türkei Vereinbarung, 18.03.2016. http://http://www.unhcr.at/home/artikel/ $16997065 \mathrm{~d} 29071553 \mathrm{e} 744 \mathrm{c} 90 \mathrm{e} 5 \mathrm{ad} 7 \mathrm{f} 4 \mathrm{c} /$ unhcr-zur-eutuerkei-vereinbarung-1.html (24.05.2016).

Vielhaber, C. (1999): Vermittlung und Interesse. Zwei Schlüsselkategorien fachdidaktischer Grundlagen im Geographieunterricht. In: Vielhaber, C. (Hrsg.): Fachdidaktik kreuz und quer. Vom Vermittlungsinteresse bis zum Methodenstreit, von der Spurensuche bis zum Raumverzicht, Materialien zur Didaktik der Geographie und Wirtschaftskunde 15. Wien, 9-26.

Wallerstein, I. (1974): The Modern World-System. New York/London: Academic Press.

\section{Konkrete Unterrichtsvorschläge}

Brändli, S., M. Eser \& K. Kistler (Hrsg.) (2009): Merken, was läuft. Rassismus im Visier. Handbuch und Materialien für den Unterricht. Zürich: Verlag Pestalozzianum.

Éducation21 (Hrsg.) (o. J.): Festung Europa: notwendig oder unmenschlich? Themenfenster: Aktualität im Unterricht. http://www.education21.ch/de/node/700 (18.04.2016).

Hintermann, C. \& H. Pichler (2003): Die EU-Erweiterung bewegt die Menschen. Migrationen in einem grenzenlosen Europa. In: Geographie heute 214, 8-11.

Jud, S. \& C. De Maddalena (2016): Ideenset Flucht und Asyl. Bern. http://www.phbern.ch/ideenset-flucht-undasyl.html (18.04.2016).
Kusturica, N. (2009): little alien. Mobilefilm. Österreich. Dvd.

Kusturica, N. (2009): Little Alien. Schulmaterialien zum Kino-Dokumentarfilm. http://www.littlealien.at/presse/ LittleAlienSchulmaterialien.pdf (18.04.2016).

Linksammlung Flucht \& Asyl. In: Journal für Politische Bildung 2/2016, 85-88.

Markom, C. \& H. Weinhäupl (2014a): Arbeitsmigration und Zugehörigkeiten. In: GW-Unterricht 133, 27-37. http://www.gw-unterricht.at/images/ pdf/gwu_133_027_037_markom_weinhaeupl.pdf (12.04.2016).

Markom, C. \& H. Weinhäupl (2014b): Migration in Bildern: Visuelle Repräsentation von Menschen mit Migrationsbiographien in Schulbüchern. In: GWUnterricht 134, 29-41. http://www.gw-unterricht.at/ images/pdf/gwu_134_029_041_markom_weinhaeupl. pdf (12.04.2016).

Markom, C. \& H. Weinhäupl (2014c): Von „Ausländer/in“ bis „Schwarzafrika“. Begriffskritiken für den Unterricht. In: GW-Unterricht 135, 42-52. http://www.gwunterricht.at/images/pdf/gwu_135_042_052_markom_ weinhaeupl.pdf (12.04.2016).

Markom, C. \& H. Weinhäupl (2014d): Migration als „Problem" in Schulbüchern und anderen Medien. In: GWUnterricht 136, 39-46. http://www.gw-unterricht.at/ images/pdf/gwu_136_39_46_markom_weinhaeupl.pdf (12.04.2016).

Mecheril, P; Melter, C.; Messerschmidt, A. \& A. de Velho (2015): Für solidarische Bildung in der globalen Migrationsgesellschaft. Ein Aufruf aus Erziehungswissenschaft, Pädagogik und Sozialer Arbeit. http://www.aufruf-fuersolidarische-bildung.de (17.06.2016)

Migration on Tour. Wanderausstellung und interaktive Online-Ausstellung mit umfangreichen Begleit- und Unterrichtsmaterialien für LehrerInnen. http://www.migrationontour.at/ (10.04.2016).

Nachrichtendienst des Bundes NDB (2016): Sicherheit Schweiz. Lagebericht 2016 des Nachrichtendienstes des Bundes. http://www.vbs.admin.ch/internet/ vbs/de/home/documentation/publication/snd_publ. parsys.41649.downloadList.49310.DownloadFile.tmp/ ndbsicherheitschweiz2016d.pdf (01.06.2016).

Padberg, S. (2012): Illegalisiert in Deutschland. Wie sähe mein Leben ohne Papiere aus? In: GW-Unterricht 128, 18-27. http://www.gw-unterricht.at/images/pdf/ gwu_128_018_027_padberg.pdf (28.05.2016).

Pichler, H. (2010a): Wo kommt meine Familie her? Spurensuche zur Erforschung der Migrationsgeschichten in der Klasse. Didaktische Konzeption - Migrationen begreifbar machen. In: Diendorfer, G., I. Ecker. H. Pichler, G. Tanzer (Hrsg.): Migration - ein Thema im Unterricht. Begleitmaterial zur interaktiven Wanderausstellung Migration on Tour. http://www. demokratiezentrum.org/fileadmin/media/pdf/MoT/ Materialien/05_Pichler_UB_WokommtFamilieher.pdf (10.04.2016).

Pichler, H. (2010b): Migration macht Geographie(n). Kooperatives offenes Lernen im Spannungsfeld Indivi- 
dualisierung und Kooperation. In: Geografie heute 285, 24-28.

Pichler, H. (2012): Migrationen und ethnische Ökonomien finden Stadt. Trittsteine für projektorientierten Unterricht vor Ort. In: Dabringer M. \& A. Trupp (Hrsg.): Wirtschaften mit Migrationshintergrund. Zur soziokulturellen Bedeutung ethnischer Ökonomien in urbanen Räumen. Innsbruck/Wien/Bozen: Studienverlag, 107119.

Sucher, J. (2016): Praxis internationaler Jugendbildung. Das Thema „Flucht, Migration und Willkommenskultur“. In: Journal für Politische Bildung 2/2016, 48-54.

Thommen, A. (2013). Neuland. Dokumentarfilm. FAMA Film AG, Züricher Hochschule der Künste, SRF. Dvd. Begleitmaterial. http:/www.filmeeinewelt.ch/deutsch/
pagesnav/framesE4.htm?../pagesmov/52087.htm\&KA (28.05.2016).

Zentrum Polis - Politik Lernen in der Schule (Hrsg.) (2013): Flucht und Migration. In: Polis aktuell, 4. http://www. politik-lernen.at/site/gratisshop/shop.item/106246.html (12.04.2016).

Zentrum Polis - Politik Lernen in der Schule (Hrsg.) (2015): Flucht und Asyl. In: Polis aktuell, 5. http://www. politik-lernen.at/site/gratisshop/shop.item/106369.html (12.04.2016).

Zentrum Polis - Politik Lernen in der Schule (Hrsg.) (2016): Transkulturelles und Interkulturelles Lernen. In: Polis aktuell, 2. http://www.politik-lernen.at/site/gratisshop/shop.item/106374.html (12.04.2016). 